\title{
Image Guided Targeted Hyperstimulation Analgesia is Superior to Placebo in Chronic Low Back Pain
}

\section{To the Editor:}

Myofascial trigger points (MTrPs) are common in people with musculoskeletal pain and may play a role in chronic nonspecific low back pain (CLBP) (1).

Nervomatrix Soleve $®$ (Fig. 1) is a novel automated robotic system that detects MTrPs by using two dimensional skin resistance measurements and subsequently applying Electric Hyperstimulation Analgesia (EHA) treatment on the detected MTrPs. EHA is claimed to provide an analgesic effect by changing the biochemical environment of MTrPs in order to reduce the primary source of nociception (1,2-4). This EHA neurostimulation is based on the physiological understanding that biochemical changes in MTrPs $(1,2,5)$ result in autonomic reactions such as increased secretion from sweat glands and ducts, which leads to variations in skin resistance (6).

In a randomized study performed by Nijs Group (1) at the University Hospital of Brussels, Belgium, a pla- cebo-treatment control group ( $\mathrm{N}=20$ ) which received six sessions of a sham procedure with Nervomatrix Soleve ${ }^{\circledR}$, applied without electric stimulation for 3 weeks in the lumbosacral region was compared with an experimental group $(\mathrm{N}=8)$ receiving 6 sessions of active EHA for 3 weeks in the same region. The experimental group included only patients for whom the Nervomatrix device was adequately measuring the impedance for pain localization; these results (Fig. 2a) were reanalyzed and presented by Nijs group.

The evolution in current pain intensity was analyzed at baseline, and at each VAS-score obtained after each of the 6 treatment sessions,

\section{Results}

VAS pain: A significant Time $x$ Group interaction was found for VAS-pain $(F(5,117)=2.708, P<.05)$. This interaction is presented in Fig. 2. Following this interaction, a simple-effects analysis revealed that pain levels

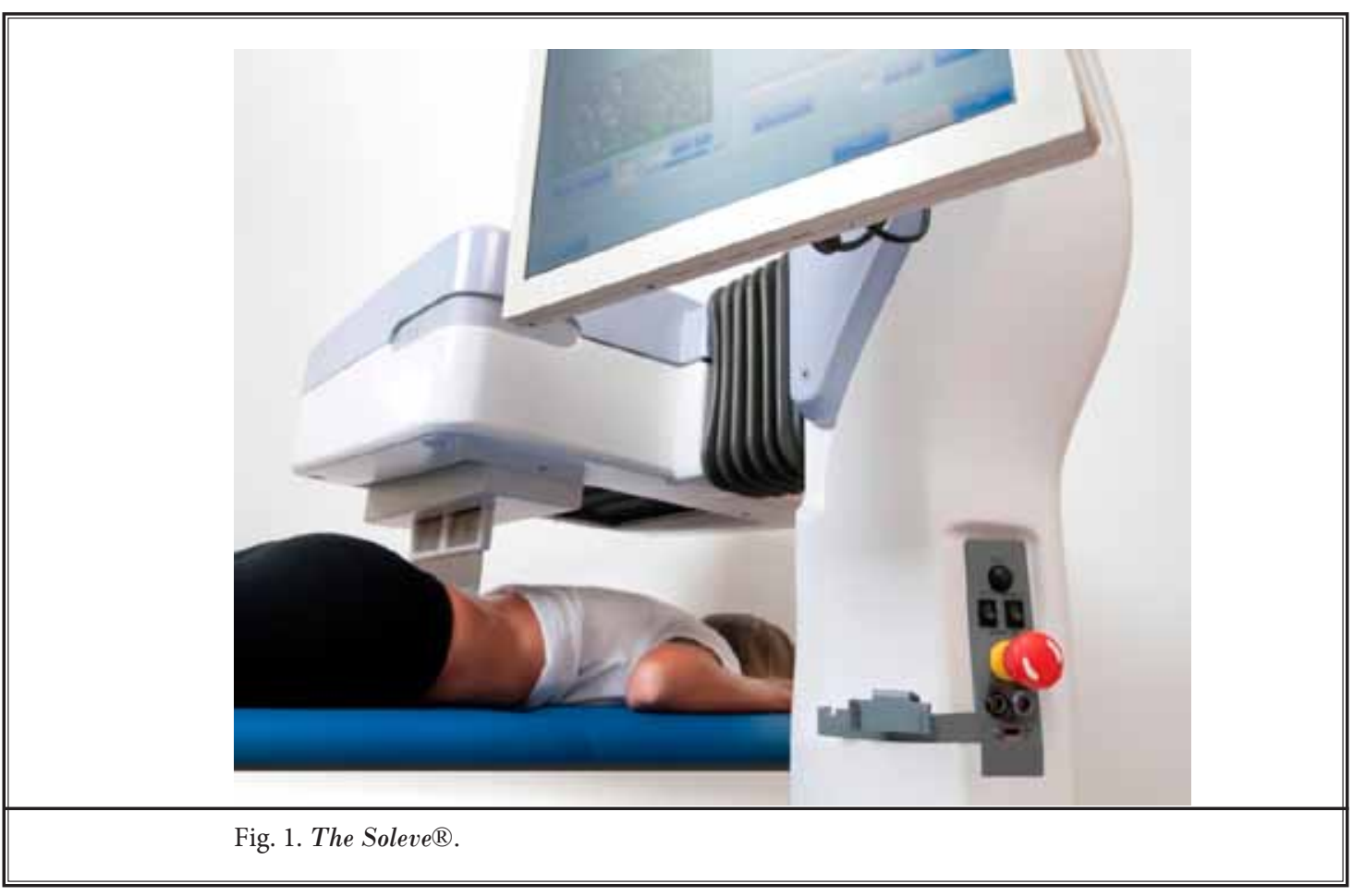




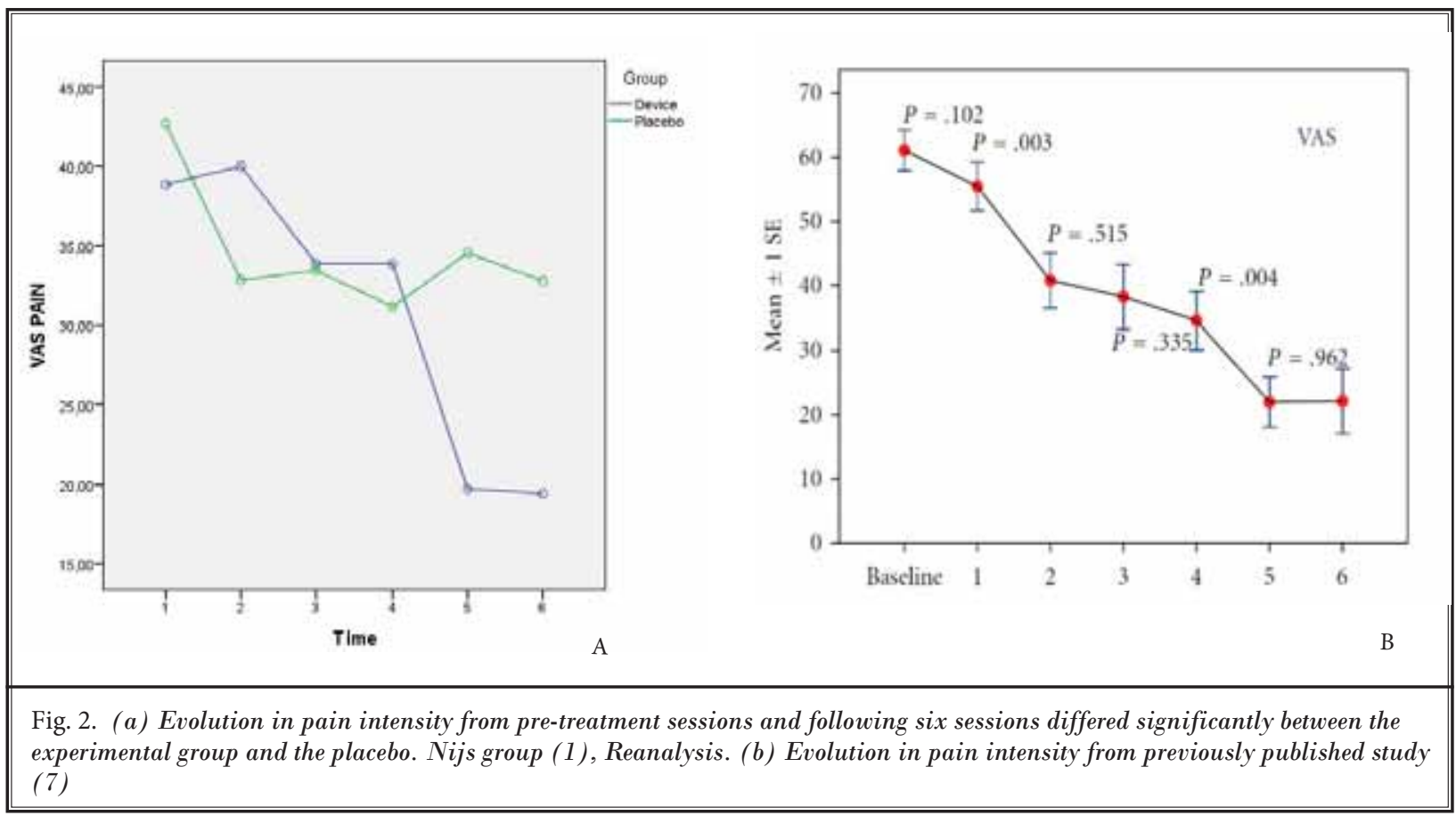

of patients in the experimental group were significantly lower before session $5(P=.05)$ but not lower before session $6(P>.05)$.

The article of Nijs published in this Journal (1) did not include the reanalysis of the data which the same authors performed subsequently when 11 patients from the experimental group for whom impedance measurements were inadequately performed are excluded. It is noted that the reanalysis conducted by Nijs Group was based on a small sample size. However, it would have been expected that these findings be fully presented and discussed within the study report published (1), and call for further investigation prior to any determination.

It is clearly demonstrated that in the desired scenario, whereby treatment is performed based on an adequate impedance matrix, EHA with Nervomatrix is superior to placebo. Interestingly, a comparison of the above presented findings with a previously published study featuring the same technology (7), showed similar findings (Fig. 2b).
Conclusions: We must be very cautious due to the small number of patients in this reanalysis. Looking at pain levels, the new device seems to reduce self-reported pain by approximately $50 \%$ in the experimental group, compared to the placebo treatment control group. Further research is required with a larger sample and with patients having either a placebo or experimental pain reduction (via the device) but only when the device is adequately functioning in its pain localization.

\section{Miguel Gorenberg, MD \\ Bnai Zion Medical Center, Nervomatrix Co-Founder \\ Haifa, Israel \\ E-mail: miguel.gorenberg@b-zion.org.il}

Ori Kanner

CEO, Nervomatrix, Israel

Kfar Haroeh, Israel

E-mail: orik@nervomatrix.com 


\section{References}

1. Aguilar Ferrándiz E, Nijs J, Gidron Y, Roussel N, Vanderstraeten R, Van Dyck D, Huysmans E, De Kooning M. AutoTargeted Neurostimulation Is Not Superior to Placebo in Chronic Low Back Pain: A Fourfold Blind Randomized Clinical Trial. Pain Physician 2016; 19:E707-E719.

2. Shah JP, Danoff JV, Desai MJ, Parikh S,Nakamura LY, Phillips TM, Gerber LH. Biochemicals associated with pain and inflammation are elevated in sites near to and remote from active myofascial trigger points. Archives of Physical Medicine and Rehabilitation 2008; 89:16-23.

3. Ballyns JJ, Shah JP, Hammond J, Gebre-
abT, Gerber LH, Sikdar S. Objective sonographic measures for characterizing myofascial trigger points associated with cervical pain. J Ultrasound Med 2011; 30:1331-1340.

4. Turo D, Otto P, Shah JP, Heimur J, Gebreab T, Zaazhoa M, Armstrong K, Gerber LH, Sikdar S. Ultrasonic characterization of the upper trapezius muscle in patients with chronic neck pain. Ultrason Imaging 2013; 35:173-187.

5. Lluch-Girbes E Nijs J, De Kooning $M$, Van Dyck D, Vanderstraeten R, Struyf F, Roussel NA. Prevalence, incidence, localization and pathophysiology of myofas- cial trigger points in patients with spinal pain: A systematic literature review. J Manipulative Physiol Ther 2015; 38:587-600.

6. Shultz SP, Driban JB, Swanik CB. The evaluation of electrodermal properties in the identification of myofascial trigger points. Arch Phy Med Rehabil 2007; 88:780-784.

7. Gorenberg M, Schiff E, Schwartz K, Eizenberg E. A novel image-guided, automatic, high-intensity neurostimulation device for the treatment of nonspecific low back pain. Pain Res Treat 2011; 2011:152307.

\section{Response: Auto-Targeted Neurostimulation In Chronic Low Back Pain: Why Available Evidence Rejects Its Clinical Utility}

\section{Dear Editor:}

Thank you for giving us the opportunity to respond to the invalid arguments raised by the company addressing our published study that examined the effectiveness of their device for treating chronic low back pain (1). Below we reply to the issues raised, showing that the company's claim regarding the effectiveness of the device is based on invalid data, attempts to change the study protocol a posteriori and hence to fraud the study in favor of the company's interest.

The company claims that they are able to tell from analyzing the 'scans' of the Soleve device that the placement of the device in some of the patients was improper. This was something that the company raised after they were informed that the clinical trial showed that the Soleve device was not superior over placebo (1). Importantly, we reported a clinical trial in the manuscript. A clinical trial reports on the effects of the treatment how it is used in clinical practice. We applied the device as it was used in clinical practice at the time the study took place, and neither the researchers, nor the therapists providing the treatment were able to verify how the company obtained those 'scans' (or what exactly the scans were telling us). Our research staff performing the study, was trained and supervised in an ongoing manner by the company, and hence, this claim could have been brought to our attention at a much earlier stage, if valid! Also, it remains unclear how those 'scans' were obtained, what type of information the scans provide, whether the scans generate valid data, or what exactly the scan findings imply. Moreover, no one of our research team ever saw any of those scans. Even if those 'scans' exist and generate valid data, it is clear that therapists using the Soleve device in clinical practice will not be able to access them in real time. Hence, therapists will not be able to use those scans to improve the treatment.

After being informed about the negative study outcome, the company had ample time to provide more information regarding those scans prior to the publication of the manuscript, but never did so. At best, those scans can be the basis for further research in this area. In their Letter to the Editor, Gorenberg and Kanner claim to provide data from such additional analysis. However, they report data from a subgroup analysis performed on the preliminary dataset of no more than 8 (out of 